\title{
Planar scanning measurement of monostatic/bistatic RCS by near-field far-field transformation based on fast multipole method
}

\author{
Shuntaro Omi ${ }^{1 \mathrm{a})}$, Toru Uno ${ }^{1}$, Takuji Arima ${ }^{1}$, Yujiro Kushiyama ${ }^{1}$, \\ and Takao Fujiii ${ }^{2}$ \\ ${ }^{1}$ Graduate School of Engineering, Tokyo University of Agriculture and Technology, \\ 2-24-16 Nakamachi, Koganei-shi, Tokyo 184-8588, Japan \\ ${ }^{2}$ Fujitsu System Integration Laboratories Ltd., \\ 4-5-6 Kamikodanaka, Nakahara-ku, Kawasaki-shi, Kanagawa 211-8588, Japan
} a)50014645205@st.tuat.ac.jp

\begin{abstract}
Radar cross section (RCS) is one of important EM properties of radar targets. Its measurement requires long distance to obtain far-field characteristics in higher frequency range such as X-band. Planar scanning near-field measurement combined with near-field far-field (NF-FF) transformation is realized by simple measurement system. However, scan area truncation of planar scanning often cause intolerable error for predicted RCS. In this paper, the accuracy of NF-FF transformation technique based on fast multipole method (FMM) with scan area of planar scanning is investigated. Keywords: RCS, near-field far-field transformation, fast multipole method Classification: Antennas and Propagation
\end{abstract}

\section{References}

[1] D. G. Falconer, "Extrapolation of near-field RCS measurements to the far zone," IEEE Trans. Antennas Propag., vol. 36, no. 6, pp. 822-829, Jun. 1988. DOI:10. $1109 / 8.1184$

[2] C. H. Schmidt, M. M. Leibfritz, and T. F. Eibert, "Fully probe-corrected nearfield far-field transformation employing plane wave expansion and diagonal translation operators," IEEE Trans. Antennas Propag., vol. 56, no. 3, pp. 737746, Mar. 2008. DOI:10.1109/TAP.2008.916975

[3] A. D. Yaghjian, "An overview of near-field antenna measurements," IEEE Trans. Antennas Propag., vol. 34, no. 1, pp. 30-45, Jan. 1986. DOI:10.1109/ TAP.1986.1143727

[4] A. C. Newell, "Error analysis technique for planar near-field measurements," IEEE Trans. Antennas Propag., vol. 36, no. 6, pp. 754-768, June 1988. DOI:10.1109/8.1177 


\section{Introduction}

Radar cross section (RCS) is one of important characteristics of radar for vehicles or aircrafts. Its measurement often requires long measurement range and huge equipment. Near-field far-field (NF-FF) transformation is a powerful approach to avoid the requirement. Falconer proposed a transformation technique for monostatic RCS measurement [1]. It enables us to predict target's monostatic RCS from only monostatic near-field (NF) measurement. However, the method is based on physical optics, so this assumption fails for some structures such as corner reflector. In such case, NF-FF transformation for antenna measurement is effective. For accurate monostatic/bistatic RCS measurement of arbitrary structure, the antenna under test (AUT) is replaced with the target illuminated by plane wave and scattered EM field is measured. An NF-FF transformation technique is proposed in ref. [2] which is based on fast multipole method (FMM) and predicts far-field of AUT by solving integral equation. This method is valid for arbitrary NF measurement grids and probes. In addition, it is more robust for NF scan area truncation relatively to conventional method [3]. Here, the planar scanning is quite convenient measurement system in terms of measurement equipment in actual measurement situation. However, the scan area truncation often introduces intolerable error. In this paper, we investigates accuracy of planar scanning measurement for RCS with scan area.

\section{Planar scanning for RCS measurement}

Fig. 1(a) shows the measurement system of planar scanning. Tx antenna transmits the incident wave and Rx antenna receives the scattered field on planar surface. The analysis of error for planar NF-FF transformation technique was described [4]. Planar scan area truncation causes two effects of the error. One of which is occurred outside of the reliable region. In this criteria, the calculated far-field is valid only in angular region $\left[-\theta_{s}, \theta_{s}\right]$ defined by target and scan area edge (see Fig. 1(b))

$$
\theta_{s}=\tan ^{-1}\left(\frac{L_{s}-d}{2 x_{0}}\right) \text {. }
$$

The other error occurs even inside the reliable region and is caused by zero assumption outside the scan area. This assumption exists in conventional method [3] and FMM based NF-FF transformation we employ is free for this error. In this paper, we investigate this error inside the reliable region and show the accuracy of planar scanning measurement with FMM based NF-FF transformation.

\section{Numerical study}

\subsection{Method}

In this paper, FMM based NF-FF transformation technique is used. So we explain this technique briefly. The Rx probe's output voltage $U$ located on $\boldsymbol{r}_{M}$ is represented by FMM formulation as

$$
U\left(\boldsymbol{r}_{M}\right)=-j \frac{\omega \mu}{4 \pi} \oiint T_{L}\left(\hat{k}, \hat{r}_{M}\right) \tilde{\boldsymbol{w}}^{*}(\hat{k}) \cdot(\overline{\boldsymbol{I}}-\hat{k} \hat{k}) \cdot \tilde{\boldsymbol{J}}(\hat{k}) d \hat{k}^{2}
$$




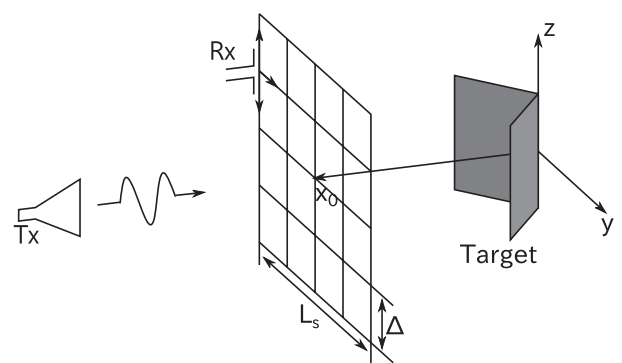

(a) Measurement system for planar scanning.

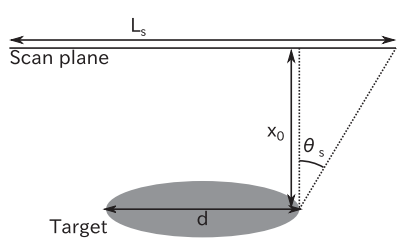

(b) The reliable region $\theta_{s}$.

Fig. 1. Measurement system and the reliable region.

where $\tilde{\boldsymbol{J}}(\hat{k})$ is the spectral representation of current density. $\tilde{\boldsymbol{w}}^{*}(\hat{k})$ is determined from probe's directivity and antenna factor. $T_{L}\left(\hat{k}, \hat{r}_{M}\right)$ is the translation operator of FMM and calculated by

$$
T_{L}\left(\hat{k}, \hat{r}_{M}\right)=-\frac{j k}{4 \pi} \sum_{l=0}^{L}(-j)^{l}(2 l+1) h_{l}^{(2)}\left(k r_{M}\right) P_{l}\left(\hat{k} \cdot \hat{r}_{M}\right)
$$

where $L$ is the multipole order. $h_{l}^{(2)}$ is the spherical Hankel function of second kind and $P_{l}$ Legendre polynomial. In eq. (2), $(\overline{\boldsymbol{I}}-\hat{k} \hat{k}) \cdot \tilde{\boldsymbol{J}}(\hat{k})$ is thought to be outgoing plane wave from the target and the translation operator translate it to incoming plane wave to the probe. So the equation represents the probe's output voltage by superposition of incoming plane wave weighted by probe's directivity $\boldsymbol{w}^{*}(\hat{k})$. By solving linear equation formulated from eq. (2), the plane wave coefficient $(\overline{\boldsymbol{I}}-\hat{k} \hat{k}) \cdot \tilde{\boldsymbol{J}}(\hat{k})$ is calculated and electric far-field is directly determined by the equation as

$$
\boldsymbol{E}(\boldsymbol{r})=-j \frac{\omega \mu}{4 \pi} \frac{e^{j k r}}{r}(\overline{\boldsymbol{I}}-\hat{r} \hat{r}) \cdot \tilde{\boldsymbol{J}}(\hat{r}) .
$$

It is noted that there is no assumption of NF outside the measured region in this method.

\subsection{Numerical results}

In order to investigate the accuracy of planar scanning RCS measurement, monostatic RCS of $2 \lambda \times 2 \lambda$ perfect electric conductor (PEC) plate is obtained by the FMM based NF-FF transformation. The NF of PEC plate is calculated by using FDTD method. After that, the calculated NF data is applied to FMM based NF-FF transformation to calculate the monostatic RCS. Observed frequency is $3[\mathrm{GHz}]$. The PEC plate is located on $y z$ plane and square scan plane is placed at $x_{0}=2.7 \lambda$. Sampling interval $\Delta=1 / 5 \lambda$. The incident wave is $-x$ directed and $z$ polarized plane wave.

The result is compared with numerical solution (reference) and the transformation result by conventional transformation technique [3]. The numerical solution is calculated from electric/magnetic current on the surface enclosing the target by using FDTD method. The conventional method is that modal coefficients are calculated from 2-D Fourier transform of the electric field on the scan plane 


$$
\overline{\boldsymbol{T}}_{t}\left(k_{y}, k_{z}\right)=\frac{e^{-j \alpha x_{0}}}{2 \pi} \iint_{-\infty}^{\infty} \boldsymbol{E}\left(x_{0}, y, z\right) e^{-j k_{y} y} e^{-j k_{z} z} d y d z
$$

where $\alpha=\sqrt{k^{2}-k_{y}^{2}-k_{z}^{2}} \cdot k$ is the wavenumber. It is noted that the effect of probe is ignored here for simplicity. Electric field in far region is calculated simply from the modal coefficients in Eq. (5). In practice, integration in eq. (5) is done in finite scan area and electric field outside the area is assumed to be zero. That introduce the truncation error inside the reliable region described in previous section.

Fig. 2 shows the error of predicted monostatic RCS for different scan length $L_{s}$. The error is calculated from below equation

$$
\Delta \sigma[\mathrm{dB}]=\sigma_{r}[\mathrm{dBsm}]-\sigma_{m}[\mathrm{dBsm}]
$$

where $\sigma_{m}$ is predicted RCS by the transformation techniques and $\sigma_{r}$ is reference value. The errors of two transformation techniques are shown. In this case, monostatic RCS is inside the reliable region for all scan length, therefore, $\Delta \sigma$ is the error inside the reliable region. As we can see, error of conventional method glows larger along the scan area reduction. The error of FMM based NF-FF transformation fall into $\pm 1.0[\mathrm{~dB}]$ for all scan length while that of conventional method exceed when $L_{S} / \lambda \leq 3$. From these results, we can conclude that FMM based NF-FF transformation is more robust for scan area truncation than conventional method.

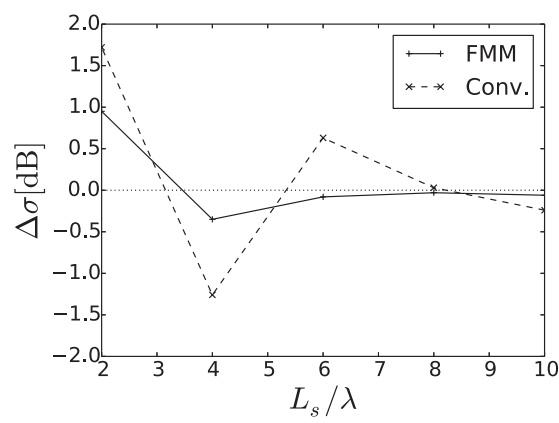

Fig. 2. Monostatic RCS error of two transformation techniques. The $\mathrm{x}$-axis indicates the length of scan area $L_{s}$ and y-axis the error in $[\mathrm{dB}]$.

\section{Experimental study}

In this section, the FMM based NF-FF transformation is applied to the actual measured NF data. Measured target is dihedral corner reflector made by bending square copper plate with $5 \lambda$ side length. The target's corner is located along $z$ axis (see Fig. 1(a)). Incident wave is transmitted to the target by a ridged horn antenna. Scattered NF is measured by $\lambda / 2$ dipole probe. The NF is measured on $5 \lambda \times 5 \lambda$ scan plane, the measured interval $\Delta=1 / 3 \lambda$. Scan plane is placed at $x_{0}=2.7 \lambda$. Fig. 3(a) shows the result of bistatic RCS in $\theta=\pi / 2$ cut. Incident wave is $-x$ directed and $z$ polarized. In this figure, reference value is numerical solution calculated in the same way as previous section. As we can see, predicted result by the transformation technique shows good agreement with reference inside the reliable region (between 
dotted lines in Fig. 3(a)). Fig. 3(b) shows the monostatic RCS result for different incident angles $\theta$ at $\phi=0$ cut. The result is calculated by using planar near-fields of different incident angles. Predicted RCS indicates high accuracy for $0-9^{\circ}$ incident angles.

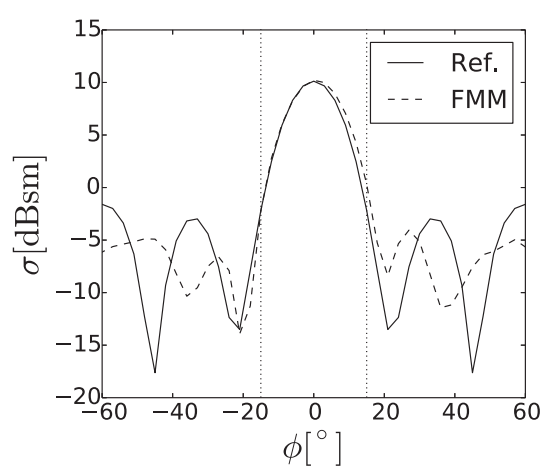

(a) Bistatic RCS in the case of $-x$ directed and $z$ polarized incident wave.

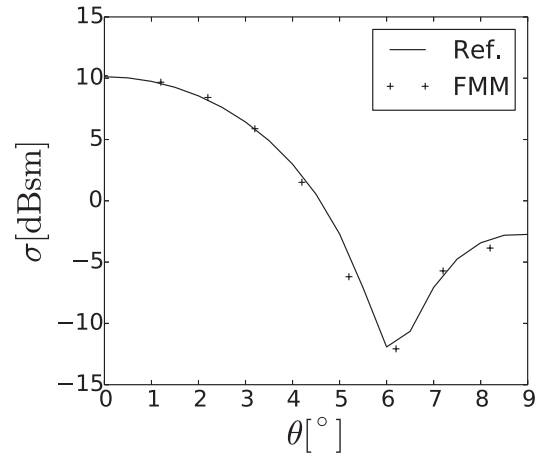

(b) Monostatic RCS.

Fig. 3. Measured RCS of dihedral corner reflector.

\section{Conclusion}

We have investigated the accuracy between the FMM based NF-FF transformation and conventional method with planar scanning to measure RCS by numerically. We have shown the robustness of FMM based NF-FF transformation technique against the scan area truncation. Also, we transformed measured NF data using FMM based NF-FF transform method. Transformed results are good agreement with reference value. By using FMM based NF-FF transformation, RCS inside reliable region is measured accurately. 